\title{
Relativistic description of heavy tetraquarks
}

\author{
D. Ebert ${ }^{1}$, R. N. Faustov ${ }^{2}$ and V. O. Galkin ${ }^{2}$ \\ 1 Institut für Physik, Humboldt-Universität zu Berlin, \\ Newtonstr. 15, D-12489 Berlin, Germany \\ 2 Dorodnicyn Computing Centre, Russian Academy of Sciences, \\ Vavilov Str. 40, 119991 Moscow, Russia
}

\begin{abstract}
The masses of the ground state and excited heavy tetraquarks with hidden charm and bottom are calculated within the relativistic diquark-antidiquark picture. The dynamics of the light quark in a heavy-light diquark is treated completely relativistically. The diquark structure is taken into account by calculating the diquark-gluon form factor. New experimental data on charmonium-like states above the open charm threshold are discussed. The obtained results indicate that $X(3872), Y(4260)$, $Y(4360), Z(4433)$ and $Y(4660)$ can be tetraquark states with hidden charm.
\end{abstract}

Recently the significant experimental progress has been achieved in heavy hadron spectroscopy. Several new charmonium-like states, such as $X(3872), Y(4260), Y(4360), Y(4660)$, $Z(4430)$, etc., were observed [1] which cannot be simply accommodated in the quarkantiquark $(q \bar{q})$ picture. These states can be considered as indications of the possible existence of exotic multiquark states [2, 3]. Here we briefly review our recent results for the masses of heavy tetraquarks in the framework of the relativistic quark model based on the quasipotential approach in quantum chromodynamics. We use the diquark-antidiquark approximation to reduce a complicated relativistic four-body problem to the subsequent more simple two-body problems. The first step consists in the calculation of the masses, wave functions and form factors of the diquarks, composed from light and heavy quarks. At the final step, a heavy tetraquark is considered to be a bound diquark-antidiquark system. It is important to emphasize that we do not consider a diquark as a point particle but explicitly take into account its structure by calculating the form factor of the diquark-gluon interaction in terms of the diquark wave functions.

In the quasipotential approach the two-particle bound state with the mass $M$ and masses of the constituents $m_{1,2}$ in momentum representation is described by the wave function $\Psi(\mathbf{p})$ satisfying the quasipotential equation of the Schrödinger type

$$
\left(\frac{b^{2}(M)}{2 \mu_{R}}-\frac{\mathbf{p}^{2}}{2 \mu_{R}}\right) \Psi_{d, T}(\mathbf{p})=\int \frac{d^{3} q}{(2 \pi)^{3}} V_{d, T}(\mathbf{p}, \mathbf{q} ; M) \Psi_{d, T}(\mathbf{q}),
$$

where the relativistic reduced mass is

$$
\mu_{R}=\frac{M^{4}-\left(m_{1}^{2}-m_{2}^{2}\right)^{2}}{4 M^{3}}
$$

and the on-mass-shell relative momentum squared

$$
b^{2}(M)=\frac{\left[M^{2}-\left(m_{1}+m_{2}\right)^{2}\right]\left[M^{2}-\left(m_{1}-m_{2}\right)^{2}\right]}{4 M^{2}} .
$$

The subscript $d$ refers to the diquark and $T$ refers to the tetraquark composed of a diquark and antidiquark. The explicit expressions for the corresponding quasipotentials $V_{d, T}(\mathbf{p}, \mathbf{q} ; M)$ can be found in Ref. [4]. 
TABLE I: Masses $M$ and form factor parameters of heavy-light diquarks. $S$ and $A$ denote scalar and axial vector diquarks antisymmetric $[Q, q]$ and symmetric $\{Q, q\}$ in flavour, respectively.

\begin{tabular}{ccccccccc}
\hline \hline \multirow{2}{*}{$\begin{array}{c}\text { Quark } \\
\text { content }\end{array}$} & $\begin{array}{c}\text { Diquark } \\
\text { type }\end{array}$ & \multicolumn{3}{c}{$Q=c$} & & \multicolumn{3}{c}{$Q=b$} \\
\cline { 3 - 5 } \cline { 7 - 8 } & $M(\mathrm{MeV})$ & $\xi(\mathrm{GeV})$ & $\zeta\left(\mathrm{GeV}^{2}\right)$ & & $M(\mathrm{MeV})$ & $\xi(\mathrm{GeV})$ & $\zeta\left(\mathrm{GeV}^{2}\right)$ \\
\hline$[Q, q]$ & $S$ & 1973 & 2.55 & 0.63 & & 5359 & 6.10 & 0.55 \\
$\{Q, q\}$ & $A$ & 2036 & 2.51 & 0.45 & & 5381 & 6.05 & 0.35 \\
{$[Q, s]$} & $S$ & 2091 & 2.15 & 1.05 & & 5462 & 5.70 & 0.35 \\
$\{Q, s\}$ & $A$ & 2158 & 2.12 & 0.99 & & 5482 & 5.65 & 0.27 \\
\hline \hline
\end{tabular}

At the first step, we calculate the masses and form factors of the light and heavy diquarks. As it is well known, the light quarks are highly relativistic, which makes the $v / c$ expansion inapplicable and thus, a completely relativistic treatment of the light quark dynamics is required. To achieve this goal we closely follow our consideration of the mass spectra of light mesons and adopt the same procedure to make the relativistic potential local by replacing $\epsilon_{1,2}(p)=\sqrt{m_{1,2}^{2}+\mathbf{p}^{2}} \rightarrow E_{1,2}=\left(M^{2}-m_{2,1}^{2}+m_{1,2}^{2}\right) / 2 M$. Solving numerically the quasipotential equation (1) with the complete relativistic potential, which depends on the diquark mass in a complicated highly nonlinear way [5], we get the diquark masses and wave functions. In order to determine the diquark interaction with the gluon field, which takes into account the diquark structure, we calculate the corresponding matrix element of the quark current between diquark states. Such calculation leads to the emergence of the form factor $F(r)$ entering the vertex of the diquark-gluon interaction [5]. This form factor is expressed through the overlap integral of the diquark wave functions. Our estimates show that it can be approximated with a high accuracy by the expression

$$
F(r)=1-e^{-\xi r-\zeta r^{2}}
$$

The values of the masses and parameters $\xi$ and $\zeta$ for heavy-light scalar diquark $[Q, q]$ and axial vector diquark $\{Q, q\}$ ground states are given in Table 【.

At the final step, we calculate the masses of heavy tetraquarks considered as the bound states of a heavy-light diquark and antidiquark. In this picture of heavy tetraquarks both scalar $S$ (asymmetric in flavour $[Q q]_{S=0}=[Q q]$ ) and axial vector $A$ (symmetric in flavour $\left.[Q q]_{S=1}=\{Q q\}\right)$ diquarks are considered. Therefore we get the following structure of the $[Q q]\left[\bar{Q} \bar{q}^{\prime}\right]$ ground $(1 S)$ states $\left(C\right.$ is defined only for $\left.q=q^{\prime}\right)$ :

- Two states with $J^{P C}=0^{++}$:

$$
\begin{aligned}
& X\left(0^{++}\right)=[Q q]_{S=0}\left[\bar{Q} \bar{q}^{\prime}\right]_{S=0} \\
& X\left(0^{++\prime}\right)=[Q q]_{S=1}\left[\bar{Q} \bar{q}^{\prime}\right]_{S=1}
\end{aligned}
$$

- Three states with $J=1$ :

$$
\begin{aligned}
& X\left(1^{++}\right)=\frac{1}{\sqrt{2}}\left([Q q]_{S=1}\left[\bar{Q} \bar{q}^{\prime}\right]_{S=0}+[Q q]_{S=0}\left[\bar{Q} \bar{q}^{\prime}\right]_{S=1}\right) \\
& X\left(1^{+-}\right)=\frac{1}{\sqrt{2}}\left([Q q]_{S=0}\left[\bar{Q} \bar{q}^{\prime}\right]_{S=1}-[Q q]_{S=1}\left[\bar{Q} \bar{q}^{\prime}\right]_{S=0}\right) \\
& X\left(1^{+-\prime}\right)=[Q q]_{S=1}\left[\bar{Q} \bar{q}^{\prime}\right]_{S=1}
\end{aligned}
$$


TABLE II: Masses of charm diquark-antidiquark ground $(1 S)$ states (in MeV). $S$ and $A$ denote scalar and axial vector diquarks.

\begin{tabular}{ccccc}
\hline \hline State & Diquark & \multicolumn{3}{c}{ Mass } \\
\cline { 3 - 5 }$J^{P C}$ & content & $c q \bar{c} \bar{q}$ & $c s \bar{c} \bar{s}$ & $c s \bar{c} \bar{q} / c q \bar{c} \bar{s}$ \\
\hline $0^{++}$ & $S \bar{S}$ & 3812 & 4051 & 3922 \\
$1^{++}$ & $(S \bar{A} \pm \bar{S} A) / \sqrt{2}$ & 3871 & 4113 & 3982 \\
$0^{++}$ & $A \bar{A}$ & 3852 & 4110 & 3967 \\
$1^{+-}$ & $A \bar{A}$ & 3890 & 4143 & 4004 \\
$2^{++}$ & $A \bar{A}$ & 3968 & 4209 & 4080 \\
\hline \hline
\end{tabular}

TABLE III: Thresholds for open charm decays and nearby hidden-charm thresholds.

\begin{tabular}{cccccc}
\hline \hline Channel & Threshold $(\mathrm{MeV})$ & Channel & Threshold $(\mathrm{MeV})$ & Channel & Threshold $(\mathrm{MeV})$ \\
\hline$D^{0} \bar{D}^{0}$ & 3729.4 & $D_{s}^{+} D_{s}^{-}$ & 3936.2 & $D^{0} D_{s}^{ \pm}$ & 3832.9 \\
$D^{+} D^{-}$ & 3738.8 & $\eta^{\prime} J / \psi$ & 4054.7 & $D^{ \pm} D_{s}^{\mp}$ & 3837.7 \\
$D^{0} \bar{D}^{* 0}$ & 3871.3 & $D_{s}^{ \pm} D_{s}^{* \mp}$ & 4080.0 & $D^{* 0} D_{s}^{ \pm}$ & 3975.0 \\
$\rho J / \psi$ & 3872.7 & $\phi J / \psi$ & 4116.4 & $D^{0} D_{s}^{* \pm}$ & 3976.7 \\
$D^{ \pm} D^{* \mp}$ & 3879.5 & $D_{s}^{*+} D_{s}^{*-}$ & 4223.8 & $K^{* \pm} J / \psi$ & 3988.6 \\
$\omega J / \psi$ & 3879.6 & & & $K^{* 0} J / \psi$ & 3993.0 \\
$D^{* 0} \bar{D}^{* 0}$ & 4013.6 & & $D^{* 0} D_{s}^{* \pm}$ & 4118.8 \\
\hline \hline
\end{tabular}

- One state with $J^{P C}=2^{++}$:

$$
X\left(2^{++}\right)=[Q q]_{S=1}\left[\bar{Q} \bar{q}^{\prime}\right]_{S=1}
$$

The orbitally excited $(1 P, 1 D \ldots)$ states are constructed analogously. As we see a very rich spectrum of tetraquarks emerges. However the number of states in the considered diquarkantidiquark picture is significantly less than in the genuine four-quark approach.

The diquark-antidiquark model of heavy tetraquarks predicts [6] the existence of the flavour $S U(3)$ nonet of states with hidden charm or beauty $(Q=c, b)$ : four tetraquarks $([Q q][\bar{Q} \bar{q}], q=u, d)$ with neither open or hidden strangeness, which have electric charges 0 or \pm 1 and isospin 0 or 1 ; four tetraquarks ( $[Q s][\bar{Q} \bar{q}]$ and $[Q q][\bar{Q} \bar{s}], q=u, d)$ with open strangeness $(S= \pm 1)$, which have electric charges 0 or \pm 1 and isospin $\frac{1}{2}$; one tetraquark $([Q s][\bar{Q} \bar{s}])$ with hidden strangeness and zero electric charge. Since in our model we neglect the mass difference of $u$ and $d$ quarks and electromagnetic interactions, corresponding tetraquarks will be degenerate in mass. A more detailed analysis [6] predicts that such mass differences can be of a few $\mathrm{MeV}$ so that the isospin invariance is broken for the $[Q q][\bar{Q} \bar{q}]$ mass eigenstates and thus in their strong decays. The (non)observation of such states will be a crucial test of the tetraquark model.

The calculated masses of the heavy tetraquark ground $(1 S)$ states and the corresponding open charm and bottom thresholds are given in Tables [IIV. We find that all $S$-wave tetraquarks with hidden bottom lie considerably below open bottom thresholds and thus they should be narrow states which can be observed experimentally. This prediction significantly differs from the molecular picture where bound $B-\bar{B}^{*}$ states are expected to lie very close 
TABLE IV: Masses of bottom diquark-antidiquark ground ( $1 S$ ) states (in MeV). $S$ and $A$ denote scalar and axial vector diquarks.

\begin{tabular}{ccccc}
\hline \hline State & Diquark & \multicolumn{3}{c}{ Mass } \\
\cline { 3 - 5 }$J^{P C}$ & content & $b q \bar{b} \bar{q}$ & $b s \bar{b} \bar{s}$ & $b s \bar{b} \bar{q} / b q \bar{b} \bar{s}$ \\
\hline $0^{++}$ & $S \bar{S}$ & 10471 & 10662 & 10572 \\
$1^{+ \pm}$ & $(S \bar{A} \pm \bar{S} A) / \sqrt{2}$ & 10492 & 10682 & 10593 \\
$0^{++}$ & $A \bar{A}$ & 10473 & 10671 & 10584 \\
$1^{+-}$ & $A \bar{A}$ & 10494 & 10686 & 10599 \\
$2^{++}$ & $A \bar{A}$ & 10534 & 10716 & 10628 \\
\hline \hline
\end{tabular}

TABLE V: Thresholds for open bottom decays.

\begin{tabular}{cccccc}
\hline \hline Channel & Threshold $(\mathrm{MeV})$ & Channel & Threshold $(\mathrm{MeV})$ & Channel & Threshold $(\mathrm{MeV})$ \\
\hline$B \bar{B}$ & 10558 & $B_{s}^{+} B_{s}^{-}$ & 10739 & $B B_{s}$ & 10649 \\
$B \bar{B}^{*}$ & 10604 & $B_{s}^{ \pm} B_{s}^{*+}$ & 10786 & $B^{*} B_{s}$ & 10695 \\
$B^{*} \bar{B}^{*}$ & 10650 & $B_{s}^{*+} B_{s}^{*-}$ & 10833 & $B^{*} B_{s}^{*}$ & 10742 \\
\hline \hline
\end{tabular}

(only few $\mathrm{MeV}$ below) to the corresponding thresholds.

The situation in the hidden charm sector is considerably more complicated, since most of the tetraquark states are predicted to lie either above or only slightly below corresponding open charm thresholds. This difference is the consequence of the fact that the charm quark mass is substantially smaller than the bottom quark mass. As a result the binding energies in the charm sector are significantly smaller than those in the bottom sector.

In Table VI] we compare our results (EFG [4]) for the masses of the ground and excited charm diquark-antidiquark bound states with the predictions of Ref. [6, 7, 8, 9] and with the masses of the recently observed excited charmonium-like states [1]. We assume that the excitations occur only inside the diquark-antidiquark bound system. Possible excitations of diquarks are not considered. Our calculation of the heavy baryon masses supports such scheme [5]. In this table we give our predictions only for some of the masses of the orbitally and radially excited states for which possible experimental candidates are available. The differences in some of the presented theoretical mass values can be attributed to the substantial distinctions in the used approaches. We describe the diquarks dynamically as quark-quark bound systems and calculate their masses and form factors, while in Ref.[6] they are treated only phenomenologically. Then we consider the tetraquark as purely the diquark-antidiquark bound system. In distinction Maini et al. consider a hyperfine interaction between all quarks which, e.g., causes the splitting of $1^{++}$and $1^{+-}$states arising from the $S A$ diquark-antidiquark compositions. From Table $\nabla 1$ we see that our dynamical calculation supports the assumption [6] that $X(3872)$ can be the axial vector $1^{++}$tetraquark state composed from the scalar and axial vector diquark and antidiquark in the relative $1 S$ state. Recent Belle and BaBar results indicate the existence of a second $X(3875)$ particle a few $\mathrm{MeV}$ above $X(3872)$. This state could be naturally identified with the second neutral particle predicted by the tetraquark model [7]. On the other hand, in our model the lightest scalar $0^{++}$tetraquark is predicted to be above the open charm threshold $D \bar{D}$ and thus to be broad, while in the model [6] it lies few $\mathrm{MeV}$ below this threshold, and thus is predicted 
TABLE VI: Comparison of theoretical predictions for the masses of the ground and excited charm diquark-antidiquark states $c q \bar{c} \bar{q}$ (in $\mathrm{MeV}$ ) and possible experimental candidates.

\begin{tabular}{|c|c|c|c|c|c|c|}
\hline \multirow{2}{*}{$\begin{array}{l}\text { State } \\
J^{P C}\end{array}$} & \multirow{2}{*}{$\begin{array}{l}\text { Diquark } \\
\text { content }\end{array}$} & \multicolumn{3}{|c|}{ Theory } & \multicolumn{2}{|c|}{ Experiment } \\
\hline & & EFG & Maiani et al. & Maiani et al. $(\operatorname{cs} \bar{c} \bar{s})$ & state & mass \\
\hline \multicolumn{7}{|l|}{$1 S$} \\
\hline $0^{++}$ & $S \bar{S}$ & 3812 & 3723 & & & \multirow{6}{*}{$\begin{array}{l}3871.4 \pm 0.6 \\
3875.4 \pm 0.7_{-2.0}^{+1.2}\end{array}$} \\
\hline \multirow{2}{*}{$1^{++}$} & \multirow{2}{*}{$(S \bar{A}+\bar{S} A) / \sqrt{2}$} & \multirow{2}{*}{3871} & \multirow{2}{*}{$3872^{\dagger}$} & & $X(3872)$ & \\
\hline & & & & & $X(3876)$ & \\
\hline $1^{+-}$ & $(S \bar{A}-\bar{S} A) / \sqrt{2}$ & 3871 & 3754 & & & \\
\hline $0^{++}$ & $A \bar{A}$ & 3852 & 3832 & & & \\
\hline $1^{+-}$ & $A \bar{A}$ & 3890 & 3882 & & & \\
\hline $2^{++}$ & $A \bar{A}$ & 3968 & 3952 & & $Y(3943)$ & $\begin{array}{l}3943 \pm 11 \pm 13 \\
3914.3_{-3.8}^{+4.1}\end{array}$ \\
\hline \multicolumn{7}{|l|}{$1 P$} \\
\hline $1^{--}$ & $S \bar{S}$ & 4244 & & $4330 \pm 70$ & $Y(4260)$ & $\left\{\begin{array}{l}4259 \pm 8_{-6}^{+2} \\
4247 \pm 12_{-32}^{+17}\end{array}\right.$ \\
\hline $1^{--}$ & $(S \bar{A}-\bar{S} A) / \sqrt{2}$ & 4284 & & & $Y(4260)$ & $4283_{-16}^{+17} \pm 4$ \\
\hline $1^{--}$ & $A \bar{A}$ & 4277 & & & & \\
\hline $1^{--}$ & $A \bar{A}$ & 4350 & & & $Y(4360)$ & $4361 \pm 9 \pm 9$ \\
\hline \multicolumn{7}{|l|}{$2 S$} \\
\hline $1^{+ \pm}$ & $(S \bar{A} \pm \bar{S} A) / \sqrt{2}$ & 4431 & & & $Z(4430)$ & $4433 \pm 4 \pm 1$ \\
\hline $0^{++}$ & $A \bar{A}$ & 4434 & & & & \\
\hline $1^{+-}$ & $A \bar{A}$ & 4461 & $\sim 4470$ & & & \\
\hline \multicolumn{7}{|l|}{$2 P$} \\
\hline $1^{--}$ & $S \bar{S}$ & 4666 & & & $Y(4660)$ & $4664 \pm 11 \pm 5$ \\
\hline
\end{tabular}

$\dagger$ input

to be narrow. Our $2^{++}$state also lies higher than the one in Ref. [6], thus making the interpretation of this state as $Y(3943)$ less probable especially if one averages the original Belle mass with the recent BaBar value wich is somewhat lower.

The recent discovery of the $Y(4260), Y(4360)$ and $Y(4660)$ indicates an excess of the expected charmonium $1^{--}$states [1]. The absence of open charm production is also inconsistent with a conventional $c \bar{c}$ explanation. Maini et al. [8] argue that $Y(4260)$ is the $1^{--} 1 P$ state of the charm-strange diquark-antidiquark tetraquark. We find that $Y(4260)$ cannot be interpreted in this way, since the mass of such $\left([c s]_{S=0}[\bar{c} \bar{s}]_{S=0}\right)$ tetraquark is found to be $\sim 200 \mathrm{MeV}$ higher. A more natural tetraquark interpretation could be the $1^{--} 1 P$ state $\left([c q]_{S=0}[\bar{c} \bar{q}]_{S=0}\right)(S \bar{S})$ which mass is predicted in our model to be close to the mass of $Y(4260)$ (see Table $\overline{V I}$ ). Then the $Y(4260)$ would decay dominantly into $D \bar{D}$ pairs. The other possible interpretations of $Y(4260)$ are the $1^{--} 1 P$ states of $(S \bar{A}-\bar{S} A) / \sqrt{2}$ and $A \bar{A}$ tetraquarks which predicted masses have close values. These additional tetraquark states could be responsible for the mass difference of $Y(4260)$ observed in different decay channels. As we see from Table VI the recently discovered resonances $Y(4360)$ and $Y(4660)$ in the 
$e^{+} e^{-} \rightarrow \pi^{+} \pi^{-} \psi^{\prime}$ cross section can be interpreted as the excited $1^{--} 1 P(A \bar{A})$ and $2 P(S \bar{S})$ tetraquark states, respectively.

Very recently the Belle Collaboration reported observation of a relatively narrow enhancement in the $\pi^{+} \psi^{\prime}$ invariant mass distribution in the $B \rightarrow K \pi^{+} \psi^{\prime}$ decay [1]. This new resonance, $Z^{+}(4430)$, is unique among other exotic meson candidates since it has a non-zero electric charge. Different theoretical interpretations were suggested [1]. Maiani et al. [9] give qualitative arguments that the $Z^{+}(4430)$ could be the first radial excitation $(2 S)$ of a diquark-antidiquark $X_{u \bar{d}}^{+}\left(1^{+-} ; 1 S\right)$ state $(A \bar{A})$ with mass $3882 \mathrm{MeV}$. Our calculations indicate that the $Z^{+}(4430)$ can indeed be the $1^{+-} 2 S[c u][\bar{c} \bar{d}]$ tetraquark state. It is the first radial excitation of the ground state $(S \bar{A}-\bar{S} A) / \sqrt{2}$, which has the same mass as $X(3872)$.

In summary, we calculated the masses of heavy tetraquarks with hidden charm and bottom in the diquark-antidiquark picture. In contrast to previous phenomenological treatments we used the dynamical approach based on the relativistic quark model. Both diquark and tetraquark masses were obtained by numerical solution of the quasipotential equation with the corresponding relativistic potentials. The diquark structure was also taken into account with the help of the diquark-gluon form factor expressed in terms of diquark wave functions. It is important to emphasize that, in our analysis, we did not introduce any free adjustable parameters but used their fixed values from our previous considerations of heavy and light meson properties. It was found that the $X(3872), Y(4260), Y(4360), Z(4433)$ and $Y(4660)$ exotic meson candidates can be tetraquark states with hidden charm. The ground states of bottom tetraquarks are predicted to have masses below the open bottom threshold and thus should be narrow.

The authors are grateful to A. Badalian, A. Kaidalov, V. Matveev, G. Pakhlova, P. Pakhlov, A. Polosa and V. Savrin for support and discussions. This work was supported in part by the Deutsche Forschungsgemeinschaft under contract Eb 139/2-4 and by the Russian Foundation for Basic Research under Grant No.05-02-16243.

[1] For recent reviews see e.g. G. V. Pakhlova, talk at the scientific session-conference of Nuclear Physics Department RAS "Physics of fundamental interactions", 25-30 November 2007, ITEP, Moscow; S. Godfrey and S. L. Olsen, arXiv:0801.3867 [hep-ph].

[2] R. L. Jaffe, Phys. Rev. D 15, 267 (1977); Phys. Rev. Lett. 38, 195 (1977); V. A. Matveev and P. Sorba, Lett. Nuovo Cim. 20, 443 (1977).

[3] A. M. Badalyan, B. L. Ioffe and A. V. Smilga, Nucl. Phys. B 281, 85 (1987); A. B. Kaidalov, Surveys in High Energy Physics 13, 265 (1999).

[4] D. Ebert, R. N. Faustov and V. O. Galkin, Phys. Lett. B 634, 214 (2006); D. Ebert, R. N. Faustov, V. O. Galkin and W. Lucha, Phys. Rev. D 76, 114015 (2007)

[5] D. Ebert, R. N. Faustov and V. O. Galkin, Phys. Rev. D 72, 034026 (2005); Phys. Lett. B 659, 612 (2008).

[6] L. Maiani, F. Piccinini, A. D. Polosa and V. Riquer, Phys. Rev. D 71, 014028 (2005).

[7] L. Maiani, A. D. Polosa and V. Riquer, Phys. Rev. Lett. 99, 182003 (2007).

[8] L. Maiani, F. Piccinini, A. D. Polosa and V. Riquer, Phys. Rev. D 72, 031502(R) (2005).

[9] L. Maiani, A. D. Polosa and V. Riquer, arXiv:0708.3997 [hep-ph]. 